
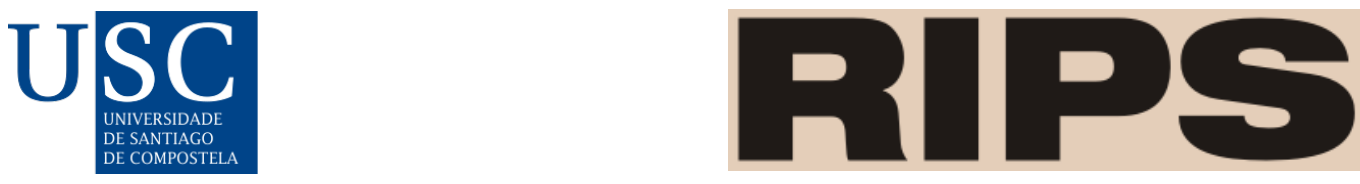

Revista

de Investigaciones

Políticas y Sociológicas

RIPS: Revista de Investigaciones Políticas y Sociológicas, 20(2), 2021. ISSN-e: 2255-5986

https://doi.org/10.15304/rips.20.2.7897

Notas de Investigación

\title{
Efectos sociales y políticos de la Covid-19 entre los estudiantes universitarios
}

Social and political effects of Covid-19 among university students

\author{
Rubén Cuéllar Rivero ${ }^{1, a}$, Araceli Mateos ${ }^{2, b}$ \\ ${ }^{1}$ Universidad de Salamanca, España \\ ${ }^{2}$ Universidad de Salamanca, España \\ a rubencuri@usal.es b amd@usal.es
}

\section{Resumen}

La pandemia provocada por el contagio masivo de coronavirus ha impactado profundamente en todos los niveles de las sociedades contemporáneas. Tratar de combatir la pandemia llevó a la mayoría de los gobiernos a imponer medidas de restricción de reuniones y movilidad, así como obligar al cumplimiento de medidas de autoprotección y de confinamiento. Esta nota de investigación presenta de manera descriptiva los resultados más relevantes obtenidos en el estudio Efectos sociales y políticos de la Covid -19 entre los estudiantes de la Universidad de Salamanca. El estudio centró su análisis en el impacto del Covid-19 en las vidas de los estudiantes de la universidad, arrojando luz sobre las emociones más habituales, el impacto psicológico, su confianza en las instituciones políticas y universitarias junto con la valoración de su gestión, además de la autoevaluación del cumplimiento de las medidas sanitarias y sus preocupaciones presentes y futuras.

Palabras clave: Covid-19; efectos; universidad; estudiantes; actitudes; opinión.

\begin{abstract}
The pandemic caused by the massive contagion of the Covid-19 virus has had a profound impact on all levels of contemporary societies. Fighting the pandemic led most national governments to impose measures to restrict meetings and mobility, as well as to force compliance with self-protection measures and collective confinements. This research note presents in a descriptive and extended way the results obtained in the study Social and political effects of Covid -19 among students at the University of Salamanca. The study focused its analysis on the impact of Covid-19 on the lives of university students, shedding light on the most common emotions, the psychological impact, their trust in political and university institutions along with the assessment of their management, in addition to the self-evaluation of compliance with sanitary measures and their present and future concerns.
\end{abstract}

Keywords: Covid-19; effects; university; students; attitudes; opinion. 


\section{INTRODUCCIÓN}

La pandemia ha obligado a la mayoría de los gobiernos a imponer medidas de contención de la expansión del nuevo coronavirus, en ocasiones limitando los derechos fundamentales de libre circulación y reunión, reduciendo la actividad económica a lo esencial y acordando confinamientos domiciliarios. Los estudiantes universitarios tuvieron que pasar, en un breve periodo de tiempo, a continuar con el curso académico confinados en sus domicilios a través de las plataformas virtuales habilitadas. Esta pandemia, y su gestión, han supuesto un impacto en la vida diaria y en las perspectivas de futuro de toda la ciudadanía. En España, tras el confinamiento domiciliario durante la primera ola de la pandemia, han sido numerosas ocasiones en las que medios de comunicación y los políticos han puesto el foco en el comportamiento social de los jóvenes y de los estudiantes a la hora de aplicar medidas de distancia social y autoprotección para combatir la propagación del virus $^{1}$.

La presente nota de investigación pretende contribuir a la incipiente literatura en Ciencias Sociales sobre el impacto multidimensional de la crisis provocada por la pandemia en las actitudes, opiniones y comportamiento de los estudiantes ante las medidas sanitarias, así como los efectos en su salud mental y en sus perspectivas sociales, académicas y laborales. Para ello, además de contextualizar teóricamente este análisis, se presentará el diseño de la investigación y el proceso de lanzamiento de la encuesta, el contexto que podía condicionar la recogida de información. Junto a ello se describen las características sociodemográficas de la muestra obtenida respecto de la población estudiantil. Finalmente, se presentan los principales hallazgos que describen el impacto del Covid-19 en la salud mental de los estudiantes, en sus perspectivas laborales y su valoración sobre la actuación de las autoridades políticas y universitarias, entre otros relevantes resultados.

\section{EL IMPACTO DEL COVID-19 EN LAS ACTITUDES POLÍTICAS Y EN EL COMPORTAMIENTO SOCIAL DE LOS ESTUDIANTES UNIVERSITARIOS}

Cada país ha tenido que abordar, en un periodo corto de tiempo, y de manera diferente la alta presión política y de incertidumbre económica, sanitaria y social vivida. Por un lado, las medidas más comunes han sido el uso obligatorio de mascarillas quirúrgicas, las medidas de distancia social o el uso de geles desinfectantes. Por otro lado, se han dado marcadas diferencias en las restricciones de aforos en espacios cerrados, las restricciones a la movilidad nocturna o interterritorial e internacional, el cierre del ocio y la hostelería o el confinamiento domiciliario. Desde las Ciencias Sociales se ha tratado de arrojar luz sobre los efectos de la pandemia en las actitudes y en el comportamiento social, especialmente, en los determinantes del grado de acuerdo y cumplimiento de las medidas de contención del virus. También se ha estudiado el impacto en la salud (física y mental) y en la situación laboral y económica de la ciudadanía y en el consecuente cambio de perspectivas vitales en el futuro próximo.

Con respecto al impacto en la salud mental de los estudiantes universitarios se han llevado a cabo distintos estudios en universidades europeas (Defeyter et al., 2021; Kohls et al., 2021; Marelli et al., 2021; Odriozola-González et al., 2020; Rodriguez-Besteiro et al., 2021) y norteamericanas (Aucejo et al., 2020; Browning et al., 2021) ${ }^{2}$ que muestran tendencias generales del alumnado a presentar cuadros de ansiedad, síntomas depresivos o altos niveles de estrés, acompañado en ocasiones de 
cierto grado de desorden alimenticio o del sueño, con marcadas diferencias por género (mayor impacto para las mujeres), situación socioeconómica o laboral, ingresos (mayor impacto en las rentas más bajas) o edad (mayor impacto en los menores de 30 años), y especialmente asociados a las restricciones, concretamente al confinamiento domiciliario o la docencia online.

Otras causas posibles del desarrollo de estos problemas de salud mental también se asocian con el impacto en las perspectivas académicas y laborales a corto y medio plazo. Específicamente, en EEUU se ha constatado la pérdida de empleo, el retraso en la graduación o las perspectivas negativas en torno a la probabilidad de encontrar trabajo después de terminar sus estudios $u$ obtener un buen salario, con diferencias según edad e ingresos personales y familiares (Aucejo et al., 2020). Un estudio realizado en 28 universidades ${ }^{3}$, en su primera ola de encuesta (llevada a cabo en los primeros meses de la pandemia), confirma de manera generalizada el impacto negativo en las perspectivas académicas y laborales de los estudiantes universitarios. En este sentido, la pandemia habría aumentado la incertidumbre de los estudiantes sobre la continuación de sus estudios o les había llevado a abandonar determinadas asignaturas, así como a cancelar prácticas formativas. Sus perspectivas laborales también se vieron menoscabadas ya que muchos de los estudiantes encuestados mostraban más disposición a trabajar mientras estudiaban o a trabajar en puestos para el que estaban sobrecualificados que antes de la pandemia. Estos patrones generales mostraban sin embargo un mayor impacto en los estudiantes con rentas paternas bajas y de sexo femenino (Jaeger et al., 2021).

El aumento de contagios y las consecutivas olas han puesto en ocasiones el foco explicativo de tal incremento en el comportamiento y la actitud de los jóvenes, lo que ha llevado a investigar cuáles son algunas de las causas del incumplimiento de las medidas y las diferencias por edad. Josep Lobera (2021) identificó algunos factores explicativos como la percepción del riesgo, los costes sociales asociados a esas medidas, el comportamiento con el entorno social y la confianza en las autoridades sanitarias. La percepción del riesgo aparece como uno de los elementos explicativos más relevantes, de hecho ésta sería más baja entre los jóvenes (Browning et al., 2021; Lobera, 2021; Olaimat et al., 2020). Con respecto a los costes sociales asociados, se destacan los de socialización y su efecto negativo en la salud mental. Derivado de ello, los jóvenes tenderían a evitar la disonancia social con el entorno, lo que favorece la relajación de las medidas de autoprotección (Lobera, 2021). Por último, la confianza en las instituciones en general, incluyendo a las instituciones sanitarias, y el mal uso de los cambios anunciados en la evidencia científica o la politización de la pandemia aparecen como factores que pudiera contribuir a disminuir la confianza entre los jóvenes (Lobera, 2021).

En este sentido, la confianza en las instituciones, concretamente en los diferentes niveles de gobierno, podría estar detrás del apoyo a las medidas de contención como el confinamiento domiciliario -de corta duración- (Bol et al., 2021). El mecanismo causal usualmente aplicado a momentos de crisis es el efecto rally round the flag que prevé un aumento de la confianza en el Gobierno y en el líder del gobierno en momento de crisis. Guglielmi et al. (2020), para el caso italiano, matizaron las proclamas que vinculan directamente mayor nivel de confianza en el Gobierno con mayor apoyo y cumplimiento de las medidas de contención del virus, añadiendo a la ecuación la confianza general en todas las instituciones, la percepción de cooperación entre ellas y la buena valoración de su labor frente a la pandemia como variables a tener en cuenta.

Algunos de estos estudios han representado el punto de partida para el diseño de esta investigación tratando de medir los efectos sociales y políticos de la Covid entre los estudiantes de 
Universidad de Salamanca. Se espera que los resultados obtenidos muestren, en consonancia con la literatura, efectos generales y efectos heterogéneos de la pandemia en función de determinadas características sociodemográficas como el sexo o la edad.

\section{DISEÑO DE INVESTIGACIÓN}

El estudio Efectos sociales y políticos de la Covid-19 entre los estudiantes de la Universidad de Salamanca (USAL) fue llevado a cabo desinteresadamente por un grupo de investigadores ${ }^{4}$ para conocer el impacto y las consecuencias de la pandemia, poniendo el foco en la vida diaria de los estudiantes universitarios ${ }^{5}$. Para ello se aplicó una encuesta online dirigida a los estudiantes de grado y posgrado de la universidad. El cuestionario fue elaborado y los datos recogidos a través del software de la plataforma Qualtrics. Se realizó un envío masivo al universo de estudiantes matriculados en el curso académico 2020-2021 vía e-mail a través del correo electrónico ${ }^{6}$ Dicho cuestionario se estructuró en 12 bloques de los cuales se describen aquí 8 que abordaban las siguientes dimensiones de interés: actitudes políticas, confianza social y confianza en instituciones, efectos del Covid-19 (emociones, malestares, y preocupaciones sociales y familiares), cumplimiento y valoración de las medidas sanitarias adoptadas, valoración de la labor de instituciones y colectivos, atribución de responsabilidad y variables sociodemográficas y de identificación política.

El universo de estudio estaba compuesto por 27018 estudiantes matriculados en el curso académico 2020-2021 en la Universidad de Salamanca, según el registro de la institución fechado en noviembre de 2020. De ellos el 16,18\% (4373) aceptaron responder la encuesta online. En la Tabla 1 puede verse la tasa de participación y la tasa de respuesta válida que, tras un proceso de validación de respuestas incompletas, spam y de encuestas con un tiempo de realización excesivamente largo o corto, arroja un 70,55\% de cuestionarios válidos con respecto a las registradas inicialmente (3085 estudiantes universitarios) ${ }^{7}$. En la Tabla 2 se muestran las principales características sociodemográficas de la muestra conseguida y de la población como el sexo, la nacionalidad, el lugar de residencia y la rama de estudios.

La encuesta fue lanzada el día 19 de enero de 2021 y conclusa el 9 de febrero de 2021. Se envió un e-mail a modo de recordatorio, volviendo a invitar a la participación el día 26 de enero de 2021. El contexto en el que se aplicó era el de un país inmerso en la tercera ola de contagios, en una universidad en el final del primer cuatrimestre y los preparativos para la realización de los exámenes de manera presencial, y una Comunidad Autónoma con medidas restrictivas como el toque de queda a las 20 horas de la tarde, limitaciones de número de personas en reuniones públicas y privadas, el cierre de establecimientos de hostelería y el cierre perimetral del territorio autonómico y provincial. 
Tabla 1. Distribución de la participación

\begin{tabular}{l|c} 
UNIVERSO DE ESTUDIO (N) & 27018 \\
\cline { 1 - 1 } RESPUESTAS REGISTRADAS & 4373 \\
\cline { 1 - 1 } TASA DE PARTICIPACIÓN & $16,18 \%$ \\
\hline RESPUESTAS VÁLIDAS Y RESPUESTAS DESCARTADAS & \\
\hline VALIDAS & 3085 \\
\hline DESCARTADAS & 1288 \\
SPAM & 36 \\
\hline INCOMPLETAS & 985 \\
\hline TIEMPO DE LA ENTREVISTA (DURACIÓN & 267 \\
INSUFICIENTE O EXCESIVA) & $70,55 \%$ \\
\hline TASA DE RESPUESTA VÁLIDA & $11,41 \%$
\end{tabular}

Fuente: Estudio Efectos sociales y políticos de la Covid -19 entre los estudiantes de la Universidad de Salamanca. 
Tabla 2. Características sociodemográficas de la muestra y de la población en la USAL

\begin{tabular}{|c|c|c|c|c|c|}
\hline $\begin{array}{l}\text { CARACTERISTICAS } \\
\text { DE LA MUESTRA }\end{array}$ & FRECUENCIA & PORCENTAJE & $\begin{array}{l}\text { CARACTERISTICAS } \\
\text { DE LA POBLACIÓN }\end{array}$ & FRECUENCIA & PORCENTAJE \\
\hline \multicolumn{3}{|l|}{ SEXO } & \multicolumn{3}{|l|}{ Sexo } \\
\hline HOMBRES & 902 & 29,2 & Hombres & 10654 & 39,4 \\
\hline MUJERES & 2151 & 69,8 & Mujeres & 16348 & 60,5 \\
\hline OTROS & 32 & 1,0 & Otros & & No disponible \\
\hline TOTAL & 3085 & 100 & Total & 27018 & 100 \\
\hline \multicolumn{3}{|l|}{ EDAD } & Edad & & \\
\hline 17-35 AÑOS & 2953 & 95,7 & & & No disponible \\
\hline MÁS DE 35 AÑOS & 132 & 4,3 & & & \\
\hline TOTAL & 3085 & 100 & Total & & \\
\hline \multicolumn{3}{|l|}{ NACIONALIDAD } & Nacionalidad & & \\
\hline ESPAÑOLA & 2829 & 91,7 & $\begin{array}{l}\text { Española } \\
\end{array}$ & 23163 & 85,7 \\
\hline OTRA & 256 & 8,3 & Otra & 3855 & 14,3 \\
\hline TOTAL & 3085 & 100 & Total & 27018 & 100 \\
\hline \multicolumn{3}{|c|}{ LUGAR DE RESIDENCIA* } & \multicolumn{3}{|l|}{ Lugar de residencia* } \\
\hline $\begin{array}{l}\text { EN LA CIUDAD DE } \\
\text { SALAMANCA }\end{array}$ & 497 & 16,1 & $\begin{array}{l}\text { En la ciudad de } \\
\text { Salamanca }\end{array}$ & 4568 & 11,4 \\
\hline $\begin{array}{l}\text { EN OTRA CIUDAD O } \\
\text { PUEBLO DE LA } \\
\text { PROVINCIA DE } \\
\text { SALAMANCA }\end{array}$ & 341 & 11,1 & $\begin{array}{l}\text { En otra ciudad o pueblo } \\
\text { de la provincia de } \\
\text { Salamanca }\end{array}$ & 3074 & 10,9 \\
\hline $\begin{array}{l}\text { EN OTRA } \\
\text { PROVINCIA DE } \\
\text { CASTILLA Y LEÓN }\end{array}$ & 900 & 29,2 & $\begin{array}{l}\text { En otra provincia de } \\
\text { Castilla y León }\end{array}$ & 6875 & 25,4 \\
\hline $\begin{array}{l}\text { EN OTRA } \\
\text { COMUNIDAD } \\
\text { AUTÓNOMA }\end{array}$ & 1132 & 36,7 & $\begin{array}{l}\text { En otra Comunidad } \\
\text { Autónoma }\end{array}$ & 10533 & 39 \\
\hline EN OTRO PAÍS & 215 & 7,0 & En otro país & 1968 & 7,3 \\
\hline TOTAL & 3085 & 100 & Total & 27018 & 100 \\
\hline $\begin{array}{l}\text { RAMA DE } \\
\text { CONOCIMIENTO }\end{array}$ & & & $\begin{array}{l}\text { Rama de } \\
\text { conocimiento }\end{array}$ & & \\
\hline $\begin{array}{l}\text { ARTES Y } \\
\text { HUMANIDADES }\end{array}$ & 717 & 23,2 & Artes y Humanidades & 4839 & 17,9 \\
\hline $\begin{array}{l}\text { CIENCIAS SOCIALES } \\
\text { Y JURÍDICAS }\end{array}$ & 1085 & 35,2 & $\begin{array}{l}\text { Ciencias Sociales y } \\
\text { Jurídicas }\end{array}$ & 10157 & 37,6 \\
\hline $\begin{array}{l}\text { CIENCIAS } \\
\text { NATURALES Y } \\
\text { MATEMÁTICAS }\end{array}$ & 427 & 13,8 & $\begin{array}{l}\text { Ciencias Naturales y } \\
\text { Matemáticas }\end{array}$ & 2820 & 10,4 \\
\hline $\begin{array}{l}\text { CIENCIAS DE LA } \\
\text { SALUD }\end{array}$ & 621 & 20,1 & Ciencias de la Salud & 5310 & 19,7 \\
\hline $\begin{array}{l}\text { INGENIERÍAS Y } \\
\text { ARQUITECTURA }\end{array}$ & 233 & 7,6 & $\begin{array}{l}\text { Ingenierías y } \\
\text { Arquitectura }\end{array}$ & 2429 & 9,0 \\
\hline TOTAL & 27018 & 100 & $\begin{array}{l}\text { NULL } \\
\text { Total }\end{array}$ & $\begin{array}{r}1463 \\
27018\end{array}$ & $\begin{array}{r}5,4 \\
100\end{array}$ \\
\hline
\end{tabular}

Fuente: Estudio Efectos sociales y políticos de la Covid -19 entre los estudiantes de la Universidad de Salamanca. *El lugar de residencia se obtuvo a partir de la agrupación de los códigos postales de los estudiantes universitarios según el registro.

\section{RESULTADOS}

A continuación, se presentan los principales hallazgos de esta investigación. Para ello, se presentan en primer lugar, los efectos generales de la pandemia entre los estudiantes, $y$, seguidamente, los efectos heterogéneos por género y edad. 


\subsection{Principales efectos sociales y políticos de la pandemia entre los estudiantes universitarios}

Con respecto a la salud mental, se pidió a los encuestados que expresaran cuál había sido la frecuencia de las emociones sentidas desde el inicio de la pandemia y la frecuencia de padecer situaciones por las que se habían sentido molestos desde el inicio de la misma. La pandemia ha despertado más emociones negativas que positivas entre los estudiantes de la universidad.

De las emociones sentidas siempre o frecuentemente por los estudiantes destacan la empatía $(77,9 \%)$, la ansiedad (61\%), el enfado $(57,2 \%)$ y el miedo $(41,9 \%)$, siendo poco frecuentes la esperanza $(25,4 \%)$ o el orgullo $(10,4 \%)$. Además, los estudiantes se habían sentido molestos siempre o frecuentemente por situaciones como sentirse nervioso/a, ansioso/a, inquieto/a (66,6\%), tener poco interés en hacer cosas (57,8\%), tener problemas para relajarse $(57,2 \%)$, sentirse decaído/ a, deprimido/a (56,4\%), no ser capaz de dejar de preocuparse ni de controlar las preocupaciones $(54,9 \%)$, siendo menos habituales las molestias por enfadarse o ponerse irritable con facilidad $(46,1 \%)$ y tener miedo, como si algo malo fuese a pasar (40,8\%). Estos resultados son consistentes con las tendencias descritas en otros estudios (Browning et al., 2021; Kohls et al., 2021; Olaimat et al., 2020; von Krakauer Hübner et al., 2020) sobre el impacto negativo de la pandemia y de algunas medidas de contención en el estado anímico y psicológico de otros universitarios.

Con respecto a las preocupaciones de los estudiantes (véase el Gráfico 1), se indagó sobre las perspectivas laborales y académicas, teniendo en cuenta otras posibles preocupaciones egotrópicas como el padecimiento personal de la Covid-19 o sociotrópicas como la preocupación por el impacto económico de la crisis sanitaria en la economía nacional. Los estudiantes mostraban, en general, alto niveles (mucho o bastante) de preocupación por todas las cuestiones planteadas, destacando el empeoramiento de la situación económica y social del país $(95,1 \%)$, la disminución de la vida social con sus familiares y amigos/as (80,7\%), la dificultad para que encuentren trabajo en un futuro próximo (76,5\%) o el empeoramiento de tu situación económica o la de su familia $(74,5 \%)$. Aunque la preocupación es menor, no dejan de ser importantes los niveles de preocupación por tener problemas para acabar este curso académico $(65,6 \%)$ y padecer personalmente problemas de salud derivados de la COVID-19 (60,7\%). Las preocupaciones por las perspectivas académicas y laborales confirman las tendencias de estudios anteriores (Aucejo et al., 2020), aunque destaca que la preocupación principal sea el devenir económico del país, posiblemente vinculado a las perspectivas socioeconómicas personales y familiares. El tipo de preocupación por la disminución de la vida social y por padecer personalmente Covid-19 es consistente con la tendencia descrita por Lobera (2021) que apunta a una importancia de la vida social en la juventud, mayoritaria entre los estudiantes, y una menor percepción del riesgo por probabilidad de contagio de Covid-19. 


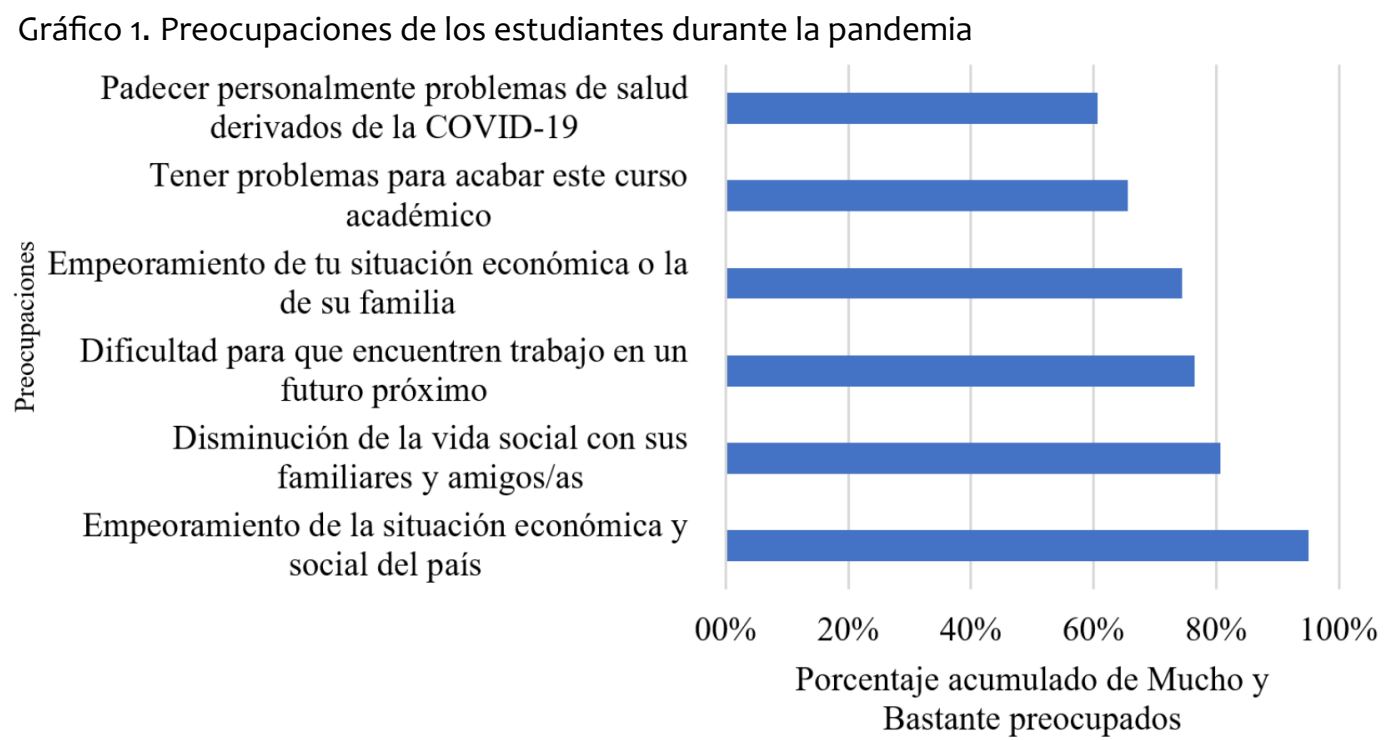

Fuente: Estudio Efectos sociales y políticos de la Covid -19 entre los estudiantes de la Universidad de Salamanca.

Por lo que se refiere a los efectos políticos, cuando se les pregunta por la conformidad general con las medidas implementadas, un 34,4\% está muy o bastante de acuerdo con ellas, frente a un $65,4 \%$ que se muestra poco a nada conforme. Este rechazo de dos terceras partes de los estudiantes encuestados contrasta con el grado de acuerdo con la necesidad de medidas más restrictivas para combatir el coronavirus (82,7\%, muy o bastante de acuerdo) y con el grado de disposición personal a hacer más sacrificios para frenar la expansión del virus (el 88,4\% afirma que probablemente sí, o sí con toda seguridad). Es posible que esta discrepancia pueda deberse al rechazo a una determinada forma de contención del virus frente a otra, o a cierto grado de deseabilidad social en pleno auge de la tercera ola en España.

El grado de implicación personal para limitar los efectos o la extensión de esta pandemia visibiliza a los estudiantes como un colectivo comprometido. Una de las más aceptadas era tener clase virtuales (77\%), el toque de queda (72,5\%) o el cierre del municipio de residencia $(68,4 \%)$. Las menos aceptadas fueron ser geolocalizado (20,2\%), la prohibición de actividades deportivas al aire libre $(40,1 \%)$ o las limitaciones de movilidad y de reuniones sociales (43,5\%). El 60,2\% aceptaría el cierre de la hostelería y el 54,2\% el confinamiento domiciliario.

Por último, los estudiantes tienen la percepción de un alto cumplimiento personal de las medidas sanitarias, tanto en el campus como en su vida diaria, (8,49 y 8,22 de media respectivamente) por encima de la valoración en la que creen que las cumplen los demás estudiantes en ambos ámbitos $(6,25$ y 5,11 de media respectivamente). Estos datos reflejan un mayor cumplimiento en el campus que en su vida diaria, quizás debido a una mayor sensación de deseabilidad social al ser una encuesta desde la propia universidad, o a una sensación de vigilancia o concienciación de las aulas como espacios cerrados.

Las valoraciones de las instituciones políticas y sociales por las que se les consulta quedan por debajo de 5 sobre 10 (siendo 0 - Muy mala y 10 - Muy Buena), y presenta correlaciones significativas con la confianza depositada en dichas instituciones. La mejor calificación la obtienen los gobiernos municipales con un 4,18, y la peor los políticos con un 2,41. El Gobierno de España y el Parlamento reciben calificaciones similares $(3,56$ y 3,58 , respectivamente $)$. 
Tabla 3. Confianza y valoración media en las instituciones por parte de los estudiantes

\begin{tabular}{|c|c|c|c|c|c|}
\hline & $\begin{array}{c}\text { CONFIANZA } \\
\text { MEDIA } \\
(0-10)\end{array}$ & $\begin{array}{l}\text { DESVIACIÓN } \\
\text { TÍPICA }\end{array}$ & $\begin{array}{c}\text { VALORACIÓN } \\
\text { MEDIA } \\
(0-10)\end{array}$ & $\begin{array}{l}\text { DESVIACIÓN } \\
\text { TÍPICA }\end{array}$ & $\begin{array}{c}\text { CORRELACIÓN } \\
\text { BIVARIADA }\end{array}$ \\
\hline $\begin{array}{l}\text { EL GOBIERNO DE } \\
\text { SU MUNICIPIO }\end{array}$ & 4,18 & 2,65 & 4,36 & 2,50 & $.699^{* *}$ \\
\hline $\begin{array}{l}\text { EL GOBIERNO DE } \\
\text { SU CCAA }\end{array}$ & 3,66 & 2,47 & 4,12 & 2,47 & $.714^{* *}$ \\
\hline EL PARLAMENTO & 3,58 & 2,38 & 3,40 & 2,23 & $.699 * *$ \\
\hline $\begin{array}{l}\text { EL GOBIERNO DE } \\
\text { ESPAÑA }\end{array}$ & 3,56 & 2,62 & 3,70 & 2,48 & $.760^{* *}$ \\
\hline $\begin{array}{l}\text { LOS MEDIOS DE } \\
\text { COMUNICACIÓN }\end{array}$ & 3,32 & 2,25 & 3,54 & 2,44 & $.695 * *$ \\
\hline $\begin{array}{l}\text { LOS PARTIDOS } \\
\text { POLÍTICOS }\end{array}$ & 2,56 & 2,13 & 2,49 & 2,07 & $.653 * *$ \\
\hline LOS POLÍTICOS & 2,41 & 2,11 & 2,58 & 2,13 & $.672 * *$ \\
\hline
\end{tabular}

Fuente: Estudio Efectos sociales y políticos de la Covid -19 entre los estudiantes de la Universidad de Salamanca

A diferencia de las instituciones sociales y políticas, las instituciones y profesionales universitarios reciben una valoración media por encima de 5. La Universidad (5,01), sus respectivas facultades $(5,62)$ o sus profesores $(6,35)$, lo que puede indicar una mayor cercanía a la institución.

Por último, se preguntó a los estudiantes sobre quienes creían los responsables de la situación actual. Tal y como muestra la Tabla 4, en primer lugar, los estudiantes identificaron a los ciudadanos que incumplen las medidas (55,4\%), en segundo lugar los políticos $(41,4 \%)$ y en tercer lugar, la responsabilidad se diluye entra diferentes respuestas pero destacan los jóvenes (22,5\%), seguidos de nadie y las autoridades sanitarias.

Tabla 4. Atribución de responsabilidad por la situación social, económica y sanitaria actual

\begin{tabular}{|c|c|c|c|}
\hline $\begin{array}{c}\text { (\% SOBRE EL TOTAL DE } \\
\text { RESPUESTAS.MULTIRESPUESTA) }\end{array}$ & $\begin{array}{l}\text { PRIMER } \\
\text { LUGAR }\end{array}$ & $\begin{array}{l}\text { SEGUNDO } \\
\text { LUGAR }\end{array}$ & $\begin{array}{l}\text { TERCER } \\
\text { LUGAR }\end{array}$ \\
\hline LOS POLÍTICOS & $31,6 \%$ & $41,4 \%$ & $15,0 \%$ \\
\hline LOS JÓVENES &, $7 \%$ & $8,2 \%$ & $22,5 \%$ \\
\hline LAS AUTORIDADES SANITARIAS & $3,3 \%$ & $12,2 \%$ & $16,4 \%$ \\
\hline LOS ESTUDIANTES UNIVERSITARIOS &, $4 \%$ & $2,2 \%$ & $4,4 \%$ \\
\hline $\begin{array}{l}\text { LOS CIUDADANOS QUE INCUMPLEN LAS } \\
\text { MEDIDAS SANITARIAS }\end{array}$ & $55,4 \%$ & $27,9 \%$ & $11,1 \%$ \\
\hline $\begin{array}{l}\text { LA DIRECCIÓN DE LAS RESIDENCIAS DE } \\
\text { ANCIANOS }\end{array}$ &, $3 \%$ & $5,2 \%$ & $10,4 \%$ \\
\hline NADIE & $8,3 \%$ & $2,9 \%$ & $20,3 \%$ \\
\hline
\end{tabular}

Fuente: Estudio Efectos sociales y políticos de la Covid -19 entre los estudiantes de la Universidad de Salamanca 


\subsection{Efectos heterogéneos de la pandemia entre los estudiantes universitarios}

\subsubsection{Género}

El impacto social y los efectos políticos de la pandemia son percibidos con mayor intensidad por el alumnado femenino que por el masculino. Con respecto al impacto en la salud mental, las estudiantes declaran haber sentido con mayor frecuencia (siempre o frecuentemente) las emociones y situaciones que les han hecho sentir más molestas desde el inicio de la pandemia que los estudiantes. En el Gráfico 2 pueden identificarse las diferencias respecto a la empatía (Mujeres 82,6\% y Hombres 67,2\%), la ansiedad (Mujeres 66,7\% y Hombres 48,2\%) y el miedo (Mujeres 48,8\% y Hombres 25,1\%) (M y $\mathrm{H}$ de ahora en adelante)). La única cuestión respecto a la que los estudiantes superan a las estudiantes es en Orgullo (9\% y 13\%, respectivamente), aunque en general ésta es una emoción poco expresada.

Gráfico 2. Emociones sentidas desde el inicio de la pandemia según género

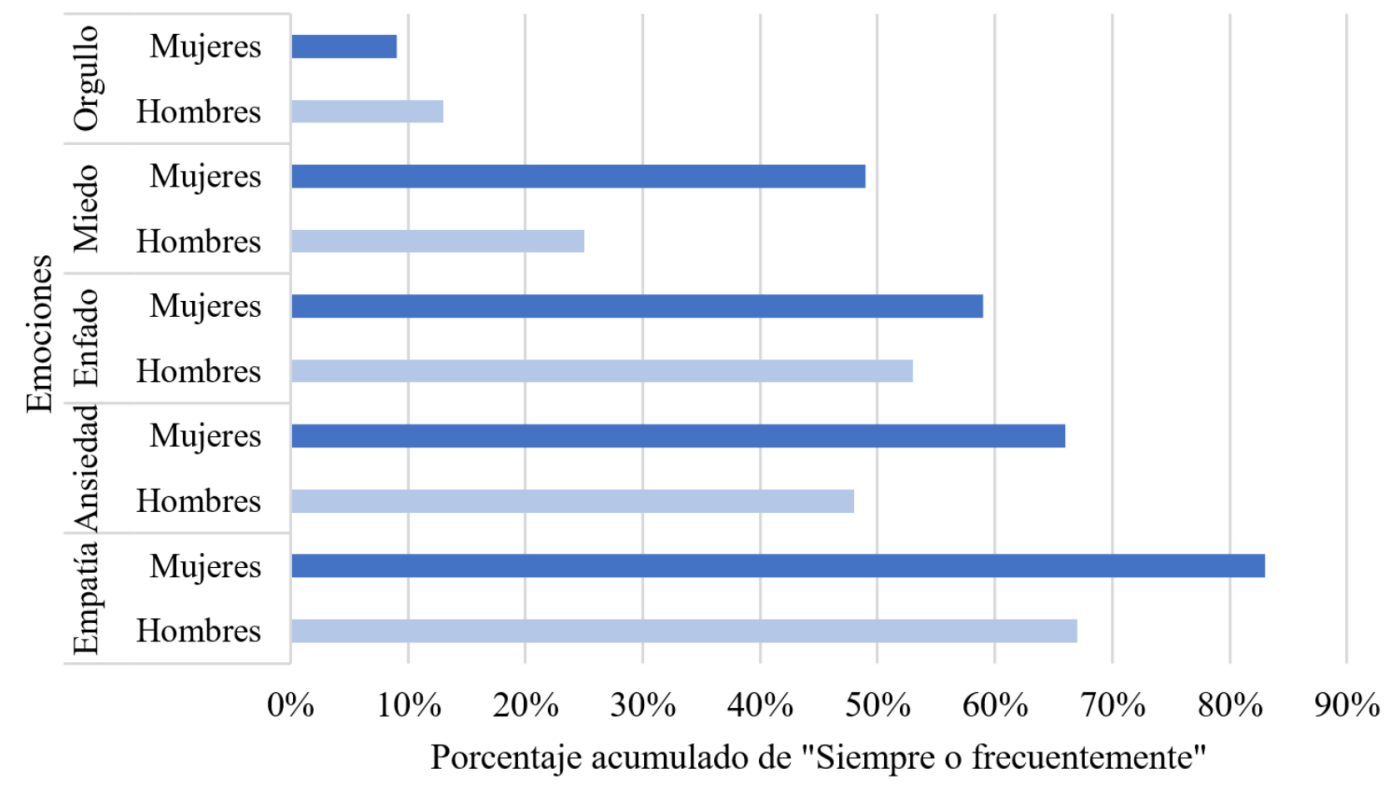

Fuente: Estudio Efectos sociales y políticos de la Covid -19 entre los estudiantes de la Universidad de Salamanca.

Con respecto a las situaciones que les habían hecho sentir molestos (siempre o frecuentemente) destacan por ser las diferencias más significativas sentirse nervioso/a, ansioso/a, inquieto/a (M: 71,5\% y H: 54,6\%), tener problemas para relajarse (M: 61,5\% y H: 46,9\%), sentirse decaído/a, deprimido/a (M: 60,2\% y H: 47,2\%), no ser capaz de dejar de preocuparse ni de controlar las preocupaciones (M: 59,8\% y H: 42,6\%), enfadarse o ponerse irritable con facilidad (M: 50,5\% y $\mathrm{H}$ : $35,4 \%$ ) y tener miedo, como si algo malo fuese a pasar (M: 44,9\% y H: 30,8\%). Estos datos dibujan un escenario de indicadores muy relevantes sobre las vivencias de los propios estudiantes y que representan elementos a tener en cuenta en futuras medidas o políticas para afrontar los efectos de la pandemia tanto desde el punto de vista institucional como universitario.

Las preocupaciones sobre las perspectivas académicas, laborales y económicas, tanto personales como a nivel nacional, aparecen con claridad diferenciadas en este estudio. Se aprecia cómo las 
estudiantes muestran más preocupación que los estudiantes por la disminución de la vida social con sus familiares y amigos/as (M: 87,8\% y H: 75,8\%), porque esta situación pueda provocar un empeoramiento de la situación económica de su familia (M: 77,1\% y H: 68,5\%) y por la dificultad para encontrar trabajo en un futuro próximo (M: 79,5\% y H: 69\%). Además, las mujeres se preocupan más por padecer personalmente problemas derivados de la Covid-19 (M: 65,9\% y H: 48,5\%) y presentan mayor preocupación por tener problemas para acabar el curso (M: 68,8\% y H: 57\%).

Los datos presentados muestran fundamentalmente la diferente percepción de los efectos negativos de la pandemia. En el ámbito psicológico y emocional las mujeres identifican un mayor número e intensidad de las emociones más negativas, así como de las situaciones que les han hecho sentir molestas. Entre estas últimas, destaca la frecuencia de sentirse molestas por nerviosismo, ansiedad e inquietud, problemas para relajarse o sentirse decaída o deprimida. Con respecto a las preocupaciones y visiones de sus perspectivas futuras, destaca la mayor preocupación por padecer problemas derivados de la Covid-19, lo que podría ser un indicio de mayor percepción del riesgo que en los hombres, y también destaca una mayor preocupación por la economía familiar o por encontrar empleo.

Estos efectos heterogéneos se aprecian en la misma medida cuando se analizan los efectos políticos: las estudiantes creen en mayor medida que los estudiantes (Muy y bastante necesario) en la necesidad de medidas para combatir el coronavirus (M: 85\% y H: 77\%) y también se muestran más dispuestas (Sí, con toda seguridad y Probablemente sí) a hacer más sacrificios en la lucha contra la Covid-19 (M: 91\% y H: 82,4\%). Sin embargo, no se han encontrado diferencias significativas respecto a qué medidas concretas a adoptar.

Las estudiantes se ven a sí mismas más cumplidoras que los estudiantes de las medidas implementadas para evitar la propagación del virus, tanto en el campus (con una media de cumplimiento de $\mathrm{M}: 8,6$ y H:8,2) como en su vida diaria (M:8,0 y H: 8,3) (Véase Tabla 5). Con respecto a estos últimos datos, estudios previos(Olaimat et al., 2020) señalaban una mayor percepción del riesgo de las mujeres, por lo que podría estar asociado con el mayor grado de acuerdo en la necesidad de más medidas de contención y en su disposición a aceptarlas. 
Tabla 5. Evaluación y autoevaluación del cumplimiento de la normativa contra el Covid-19 por parte de los estudiantes según género

\begin{tabular}{l|lccc}
\multicolumn{2}{l}{} & N & MEDIA & $\begin{array}{c}\text { DESV. } \\
\text { DESVIACIÓN }\end{array}$ \\
\hline $\begin{array}{l}\text { ESTUDIANTES EN EL } \\
\text { CAMPUS }\end{array}$ & Mujeres & 2144 & 6,22 & 2,19 \\
& Hombres & 899 & 6,33 & 2,18 \\
& Total & 3043 & 6,27 & \\
$\begin{array}{l}\text { ENCUESTADO/A EN EL } \\
\text { CAMPUS }\end{array}$ & Mujeres & 2146 & $8,62^{* * *}$ & 1,74 \\
& Hombres & 896 & $8,22^{* * *}$ & 2,05 \\
ESTUDIANTES EN SU & Total & 3042 & 8,42 & \\
VIDA DIARIA & Mujeres & 2143 & $5,05^{*}$ & 2,47 \\
& Hombres & 897 & $5,22^{*}$ & 2,47 \\
ENCUESTADO/A SU & Total & 3040 & 5,13 & \\
VIDA DIARIA & Mujeres & 2149 & $8,31 * * *$ & 1,52 \\
& Hombres & 902 & $8,00^{* * *}$ & 1,66 \\
& Total & 3051 & 8,15 &
\end{tabular}

Fuente: Estudio Efectos sociales y políticos de la Covid -19 entre los estudiantes de la Universidad de Salamanca. * Diferencia estadísticamente significativa $\mathrm{p}<0,0 .{ }^{* * *}$ Diferencia estadísticamente significativa $\mathrm{p}<0,000$.

\subsubsection{Edad}

Los resultados de este estudio también hallaron diferencias por edad. Para facilitar la lectura e interpretación de los datos, se han creado tres categorías: 18 a 23 años, 24 a 29 años y más de 30 años.

Los estudiantes menores de 30 años presentan con mayor frecuencia (siempre o frecuentemente) las emociones negativas descritas (ansiedad, miedo y enfado). Por su parte, los mayores de 30 años declaran en mayor frecuencia esperanza que los estudiantes más jóvenes. Los tres grupos de estudiantes declaran haber sentido, siempre o frecuentemente, empatía (por encima del 70\%), siendo algo mayor entre 18 a 23 años (78,3\%) y de 29 a 30 años $(78,1 \%)$ que entre el grupo de más de 30 años (72,9\%). Las diferencias en el Orgullo sentido son mínimas por edad.

Cuando se les pregunta por las situaciones que les han hecho sentir molestos, los estudiantes de 18 a 23 años son los que en mayor medida se han sentido molestos por todas las situaciones por las que se preguntaba. El Gráfico 3 muestra estas diferencias por grupos de edad: sentirse nervioso/ a, ansioso/a, inquieto/a (de 18 a 23 años, 70,3\%, de 24 a 29 años, 62,6\%, y más de 30 años, 37,6\%), sentirse decaído/a, deprimido/a (de 18 a 23 años, 60,2\%, de 24 a 29 años, 51,3\%, y más de 30 años, 29,4\%), no ser capaz de dejar de preocuparse ni de controlar las preocupaciones (de 18 a 23 años, $58,7 \%$, de 24 a 29 años, $48,2 \%$, y más de 30 años, 30,3\%), tener problemas para relajarse (de 18 a 23 años, $59,5 \%$, de 24 a 29 años, 55,4\%, y más de 30 años, 38,5\%) y de tener poco interés en hacer cosas (de 18 a 23 años, 60,8\%, de 24 a 29 años, 53,8\%, y más de 30 años, 30,6\%). 
En su conjunto, estos resultados indican un mayor impacto de la pandemia en la salud mental del grupo de estudiantes entre 18 y 23 años, frente a los mayores de 30 años. De nuevo, estos datos están en línea de otros trabajos (Marelli et al., 2021; Olaimat et al., 2020) que han estudiado el impacto de la pandemia y de las medidas de contención del virus adoptas por las autoridades en la salud mental de los jóvenes.

Gráfico 3. Situaciones que les han hecho sentirse molestos desde el inicio de la pandemia según grupos de edad

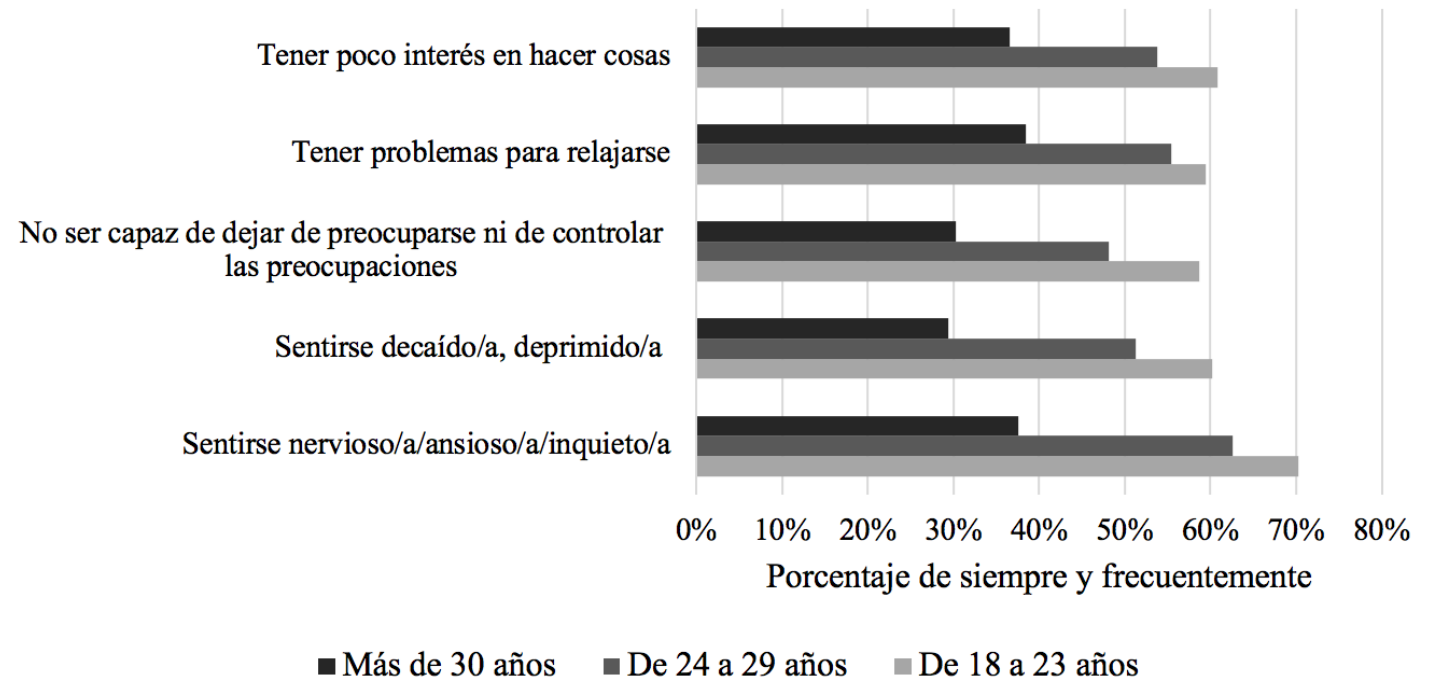

Fuente: Estudio Efectos sociales y políticos de la Covid -19 entre los estudiantes de la Universidad de Salamanca.

Cuando se pregunta por las preocupaciones sobre su salud, vida social y perspectivas académicas y laborales, los estudiantes entre 18 y 23 años y entre 24 y 29 años presentan una mayor preocupación (Mucho y bastante) por la disminución de la vida social con sus familiares y amigos/ as (82\% y 80,3\%) que el grupo de más de 30 años (67\%). Tendencia similar respecto a la preocupación por la dificultad para encontrar trabajo en un futuro próximo $(77,8 \%, 77,7 \%, 58,8 \%$, respectivamente) y la preocupación por tener problemas para acabar el curso (68,9\%, 58,8\%, $50,7 \%)$. Los tres grupos de edad tienen niveles de preocupación igual de altos porque esta situación pueda provocar un empeoramiento de la situación económica de su familia (el 74,1\% entre los de 18 y 23 años y el 76\% entre los mayores de 30 años) o por el empeoramiento de la situación económica y social del país (superiores al 95\% en los tres grupos). Los jóvenes presentan una mayor preocupación por su futuro académico, laboral, y especialmente el social, en consonancia con la literatura sobre el impacto socioeconómico de la pandemia en los estudiantes universitarios. Sin embargo, la preocupación por padecer personalmente problemas derivados de la Covid-19 se invierte en función de la edad: los mayores de 30 años declaran en mayor medida esta preocupación $(64,8 \%)$ frente a los de 18 a 23 años y de 24 a 29 años (61,2\% y 56,6\% respectivamente). Esta última diferencia muestra la distinta percepción del riesgo en el contagio, menor en los jóvenes y mayor conforme incrementa la edad.

Entrando a valorar los diferentes efectos políticos por edad, el grupo de 18 a 23 años se muestra menos conforme con las medidas sanitarias adoptadas para la contención del virus (Mucho y Bastante -33,1\%-) que el grupo de 24 a 29 años (37,8\%) o que el grupo de más de 30 años $(40,2 \%)$. Este hecho puede estar asociado a los costes sociales de estas medidas, que se ejemplifican en la disminución de la vida social. Aunque los tres grupos de edad tienen una alta disposición a asumir 
más restricciones para contener el virus, el grupo más joven y el grupo intermedio se muestran ligeramente más dispuestos (83,3\% y 82,3\% de Sí, con toda seguridad y Probablemente sí) que el grupo de más de 30 años $(77,8 \%)$. Por lo que se refiere a las medidas concretas, solo se aprecian diferencias a medida que incrementa la edad en asumir el cierre de la hostelería (58,6\% entre los de 18 a 23 años, 63,6\% entre los de 24 a 29 años, y el 69,9\% entre los de más de 30 años) o la prohibición de circular y reunirse con no convivientes (40,1\%, 49,5\%, 65,9\%, respectivamente).

En lo que respecta a la valoración que los estudiantes hacen de sí mismos y de sus iguales en relación al cumplimiento de la normativa Covid-19, la tendencia (véase la Tabla 4) muestra una mejor autoevaluación que la del resto de estudiantes, tanto dentro como fuera del campus. Si se atiendo ahora a los estudiantes en el campus, destaca que los que tienen entre 18 y 23 años, evalúan el cumplimiento de las normas que hacen sus pares mejor que la que hacen el resto de los grupos de edad de los suyos. Esto se invierte cuando los estudiantes evalúan el cumplimiento de los demás estudiantes en la vida diaria fuera del campus: los mayores de 30 años ponen la mejor calificación de sus paren con un 5,52 . 
Tabla 6. Evaluación y autoevaluación del cumplimiento de la normativa contra el Covid-19 por parte de los estudiantes según grupos de edad

\begin{tabular}{|c|c|c|c|c|}
\hline & & $\mathbf{N}$ & MEDIA & $\begin{array}{c}\text { DESV. } \\
\text { DESVIACIÓN }\end{array}$ \\
\hline \multirow{4}{*}{$\begin{array}{l}\text { ESTUDIANTES EN EL } \\
\text { CAMPUS }\end{array}$} & De 18 a 23 años & 2338 & $6,32 *$ & 2,14 \\
\hline & De 24 a 29 años & 520 & $6,02^{*}$ & 2,29 \\
\hline & Más de 30 años & 217 & 6,09 & 2,39 \\
\hline & Total & 3075 & 6,25 & 2,18 \\
\hline \multirow{4}{*}{$\begin{array}{c}\text { ENCUESTADO/A EN EL } \\
\text { CAMPUS }\end{array}$} & De 18 a 23 años & 2341 & 8,46 & 1,85 \\
\hline & De 24 a 29 años & 520 & 8,48 & 1,98 \\
\hline & Más de 30 años & 213 & $8,89 * *$ & 1,61 \\
\hline & Total & 3074 & 8,49 & 1,86 \\
\hline \multirow{4}{*}{$\begin{array}{l}\text { ESTUDIANTES EN SU } \\
\text { VIDA DIARIA }\end{array}$} & De 18 a 23 años & 2340 & 5,12 & 1,99 \\
\hline & De 24 a 29 años & 518 & 4,91 & 2,14 \\
\hline & Más de 30 años & 214 & $5,52^{* *}$ & 2,25 \\
\hline & Total & 3072 & 5,11 & 2,04 \\
\hline \multirow{4}{*}{$\begin{array}{l}\text { ENCUESTADO/A SU } \\
\text { VIDA DIARIA }\end{array}$} & De 18 a 23 años & 2341 & $8,16^{* * *}$ & 1,60 \\
\hline & De 24 a 29 años & 521 & $8,29 * * *$ & 1,51 \\
\hline & Más de 30 años & 221 & $8,63 * * *$ & 1,39 \\
\hline & Total & 3083 & 8,22 & 1,57 \\
\hline
\end{tabular}

Fuente: Estudio Efectos sociales y políticos de la Covid -19 entre los estudiantes de la Universidad de Salamanca. Se realizó una prueba ANOVA para la diferencia de medias. * Diferencia del grupo con respecto a los otros estadísticamente significativa $\mathrm{p}<0,0 .{ }^{* *}$ Diferencia del grupo con respecto a los otros estadísticamente significativa $\mathrm{p}$ $<0,00$. ${ }^{* * *}$ Diferencia del grupo con respecto a los otros estadísticamente significativa $\mathrm{p}<0,000$.

Finalmente, cuando se analiza la valoración de las instituciones y la confianza depositada en ellas por grupos de edad, con la excepción de la valoración que hacen los estudiantes según su edad de las instituciones universitarias, las diferencias de medias se muestran estadísticamente significativas. La tendencia es similar a la mostrada anteriormente obteniendo mejor valoración las instituciones más cercanas. En primer lugar, los profesores (de 18 a 23 años una nota media de 6,10, de 24 a 29 años, 6,73 y de más de 30 años, 8,09), seguido de las facultades (de 18 a 23 años una nota media de 5,34, de 24 a 29 años, 6,07 y de más de 30 años, 7,63) y de la Universidad en general (de 18 a 23 años una nota media de 5,34, de 24 a 29 años, 6,07 y de más de 30 años, 7,63). En la atribución de responsabilidades por edad, no se identifican diferencias relevantes. 


\section{CONCLUSIONES}

La pandemia y las medidas adoptadas por las autoridades políticas y académicas han tenido efectos emocionales, sociales y políticos entre los estudiantes universitarios. Los datos de la investigación aquí presentada, con una muestra amplia de estudiantes de grado, master y doctorado, ayudan a aportar evidencia empírica de las consecuencias que esta pandemia está provocando en este colectivo.

Los efectos aquí identificados muestran la misma tendencia que los señalados por otros estudios realizados en otras universidades a nivel internacional y a la población en general indicados en el marco teórico. Al mismo tiempo, dibujan un panorama heterogéneo, al menos teniendo en cuenta el sexo y la edad del alumnado.

En primer lugar, se identifica un importante impacto emocional y psicológico en los estudiantes universitarios producto de la pandemia. Haber sentido ansiedad, miedo o enfado, junto a sentirse deprimido, nervioso o ansioso, y que estas sensaciones llegaran a preocuparles de manera importante, dibuja un escenario al que es necesario prestar mayor atención. Este impacto ha sido de mayor relevancia entre las estudiantes que entre los estudiantes, y aparece asociado a la diferente vivencia de las medidas de contención del virus, las restricciones de movilidad y también con la percepción del impacto futuro en las perspectivas sociales, laborales y académicas, tanto familiares como personales.

En segundo lugar, la valoración de las perspectivas a corto o medio plazo en términos académicos, económicos y profesionales también se han visto alteradas por la pandemia. En este caso son percibidas de diferente forma por los más jóvenes (fundamentalmente preocupados por el desarrollo de su futuro académico más inmediato), que por los más mayores, para los que la mella que deja la pandemia es mucho más relevante en sus perspectivas laborales.

Por su parte, la valoración de las instituciones políticas y sociales es algo negativa, asociada a los bajos niveles de confianza depositada en dichas instituciones. Únicamente hacen una valoración positiva del ámbito más cercano: la universidad, sus facultades y su profesorado. Sin embargo, las valoraciones negativas señaladas no parecen estar totalmente asociadas a la atribución de responsabilidad de la situación vivida en el momento de aplicación de la encuesta, ya que en primer lugar se les atribuye dicha responsabilidad es a los ciudadanos que incumplen las medidas.

Siguiendo similares tendencias respecto a otros ámbitos y temas, los estudiantes tienden a realizar una autoevaluación del cumplimiento de las normas de manera más optimista que la que realizan de sus pares, tanto en el campus como en su vida diaria. Podría decirse que se trata de jóvenes que, aunque son críticos con las algunas de las medidas, presentan una actitud positiva, se autoevalúan positivamente y, al menos en el momento de aplicación de la encuesta -tercera ola-, eran proclives a asumir otro tipo de restricciones con el objetivo de paliar y reducir los contagios.

Por último, este estudio fue realizado en un momento y contexto concreto, lo que ofrece una fotografía relevante sobre los estudiantes universitarios a partir de una muestra muy amplia, si bien, la realización de otros estudios similares, en otro momento, y otras universidades, facilitará

la identificación de tendencias o cambios en los efectos sociales y políticos de la pandemia entre este colectivo de estudiantes. 


\section{Bibliografía}

AUCEJO, E. M., FRENCH, J., UGALDE ARAYA, M. P., \& ZAFAR, B. (2020). The impact of COVID-19 on student experiences and expectations: Evidence from a survey. Journal of Public Economics, 191. https://doi.o rg/10.1016/j.jpubeco.2020.104271

BOL, D., GIANI, M., BLAIS, A., \& LOEWEN, P. J. (2021). The effect of COVID-19 lockdowns on political support: Some good news for democracy? European Journal of Political Research, 60(2), 497-505. https://doi. org/10.1111/1475-6765.12401

BROWNING, M. H. E. M., LARSON, L. R., SHARAIEVSKA, I., RIGOLON, A., MCANIRLIN, O., MULLENBACH, L., CLOUTIER, S., Vu, T. M., THOMSEN, J., REIGNER, N., METCALF, E. C., D’ANTONIO, A., HELBICH, M., BRATMAN, G. N., \& Alvarez, H. O. (2021). Psychological impacts from COVID-19 among university students: Risk factors across seven states in the United States. PloS One, 16(1), e0245327. https://do i.org/10.1371/journal.pone.0245327

DEFEYTER, M. A., STRETESKY, P. B., LONG, M. A., FUREY, S., REYNOLDS, C., PORTEOUS, D., DODD, A., MANN, E., KEMP, A., FOX, J., MCANALLEN, A., \& GONÇALVES, L. (2021). Mental Well-Being in UK Higher Education During Covid-19: Do Students Trust Universities and the Government? Frontiers in Public Health, 9. $\underline{\mathrm{h}}$ ttps://doi.org/10.3389/fpubh.2021.646916

GUGLIELMi, S., DOTTI SANI, G. M., MOLTENI, F., BIOLCATI, F., CHIESI, A. M., LADINI, R., MARAFFI, M., PEDRAZZANI, A., \& VEZZONI, C. (2020). Public acceptability of containment measures during the COVID-19 pandemic in Italy: how institutional confidence and specific political support matter. International Journal of Sociology and Social Policy, 40(9-10), 1069-1085. https://doi.org/10.1108/I ISSP-07-2020-0342

JAEGER, D. A., ARELLANO-BOVER, J., KARBOWNIK, K., MARTÍNEZ-MATUTE, M., NUNLEY, J., \& SEALS, R. A. (2021). The Global COVID-19 Student Survey: First Wave Results. www.iza.org

KOHLS, E., BALDOFSKI, S., MOELLER, R., KLEMM, S.-L., \& RUMMEL-KLUGE, C. (2021). Mental Health, Social and Emotional Well-Being, and Perceived Burdens of University Students During COVID-19 Pandemic Lockdown in Germany. Frontiers in Psychiatry, 12. https://doi.org/10.3389/fpsyt.2021.643957

LOBERA, J. (2021, May 22). Los jóvenes y el cumplimiento de las medidas sanitarias. Agenda Pública.

MARELli, S., CASTELNUOVO, A., SOMMA, A., CASTRONOVO, V., MOMBELli, S., BOTTONI, D., LEITNER, C., FOSSATI, A., \& FERINI-STRAMBI, L. (2021). Impact of COVID-19 lockdown on sleep quality in university students and administration staff. Journal of Neurology, 268(1), 8-15. https://doi.org/10.1007/s0041 5-020-10056-6

ODRIOZOLA-GONZÁlEZ, P., PLANCHUElO-GÓMEZ, Á., IRURTIA, M. J., \& de LUIS-GARCÍA, R. (2020). Psychological effects of the COVID-19 outbreak and lockdown among students and workers of a Spanish university. Psychiatry Research, 290. https://doi.org/10.1016/j.psychres.2020.113108

OLAIMAT, A. N., AOLYMAT, I., ELSAHORYI, N., SHAHBAZ, H. M., \& HOLLEY, R. A. (2020). Attitudes, Anxiety, and Behavioral Practices Regarding COVID-19 among University Students in Jordan: A Cross-Sectional Study. American Journal of Tropical Medicine and Hygiene, 103(3), 1177-1183. https://doi.org/10.42 69/ajtmh.20-0418

RODRIGUEZ-BESTEIRO, S., TORNERO-AGUILERA, J. F., FERNÁNDEZ-LUCAS, J., \& CLEMENTE-SUÁREZ, V. J. (2021). Gender Differences in the COVID-19 Pandemic Risk Perception, Psychology, and Behaviors of Spanish University Students. International Journal of Environmental Research and Public Health, 18.

VON KRAKAUER HÜBNER, C., DE LIMA BRUSCATTO, M., \& LIMA, R. D. (2020). Distress among Brazilian university students due to the Covid-19 pandemic: survey results and reflections. https://doi.org/10.11 $\underline{01 / 2020.06 .19 .20135251}$ 
Notas

$1 \quad$ Sirvan de ejemplo algunos titulares como "El aumento de contagios entre jóvenes acelera la transmisión de la covid en España” de El País (https://elpais.com/sociedad/2021-06-28/el-aumento-de-contagios-entre-jovenes -acelera-la-transmision-de-la-covid-en-espana.html), "La explosión de contagios entre los jóvenes complica el verano en España” de elDiario.es (https://www.eldiario.es/sociedad/explosion-contagios-jovenes-complica-ve rano-espana_1_8095561.html) o "Los conciertos, fiestas en barcos y hoteles, los focos de contagio entre los estudiantes" de RTVE (https://www.rtve.es/noticias/20210625/conciertos-fiestas-barcos-hoteles-focos-contagi o-estudiantes/2111961.shtml).

2 Véase los trabajos de Olaimat et. Al (2020) y von Krakauer Hübner at al. (2020) para estudios en Brasil y Jordania.

3 “14 son de Estados Unidos, 5 de España, 3 de Australia, 2 de Suecia, 2 de Austria, 1 de Italia y 1 de México" (Aucejo et al., 2020).

4 Araceli Mateos (Universidad de Salamanca), Mariano Torcal (Universitat Pompeu Fabra), Manuel Alcántara (Universidad de Salamanca), Emily Carty (Universidad de Salamanca), Daniel Caro (Universidad de Salamanca) y Rubén Cuéllar (Universidad de Salamanca)

5 La base de datos de este estudio, junto con el Libro de Códigos y el cuestionario utilizado, se encuentra disponible para su libre descarga y uso en la página web del Área de Ciencia Política y Administración Pública de la Universidad de Salamanca.

6 El estudio contó con la aprobación del Comité de Bioética de la Universidad de Salamanca y del Delegado de protección de datos, así como con la colaboración del equipo de gobierno de la universidad que facilitó el mailing de estudiantes.

7 En el diseño de la encuesta, el tiempo de realización de la misma se estimaba en 10 minutos (600 segundos), por tanto, se desestimaron los cuestionarios cuya duración fuera menor o igual a 480 segundos (8 minutos). En el otro extremo, no se consideraron válidos los cuestionarios cuya duración era superior a 81.123 segundos, corte calculado a través de la media de la duración de todos los cuestionarios más tres desviaciones típicas. En total, se eliminaron el $6,11 \%$ de los cuestionarios obtenidos inicialmente.

$8 \quad$ La Universidad no ofreció un significado específico para la categoría "NULL". 